\title{
Das PRISMA-Statement - was sollte über systematische Übersichtsarbeiten berichtet werden?
}

Forschung braucht Kommunikation - innerhalb der Fachkreise und mit der Öffentlichkeit. In Studienberichten müssen Ziele, Methoden und Ergebnisse vollständig und transparent dargestellt werden, damit die Erkenntnisse sinnvoll genutzt und in der Planung zukünftiger Forschung berücksichtigt werden können. Die Ergebnisse patientenorientierter klinischer Studien sollten zudem möglichst schnell und unverfälscht in der Gesundheitsversorgung umgesetzt werden, da jede Verzögerung für Patienten vermeidbare Krankheit oder gar Tod bedeuten kann.

In den letzten 15 Jahren haben sich mehrere Initiativen formiert, um Empfehlungen für das Publizieren von Studienberichten (sog. „Reporting Guidelines“) zu erarbeiten. Gemeinsames Ziel ist die Verbesserung der Qualität und Vollständigkeit wissenschaftlicher Artikel. Als erste Empfehlung wurde 1996 das CONSORT-Statement vorgestellt, das für die Berichterstattung von randomisierten klinischen Studien gilt. Nach vollständiger Revision im Jahr 2001 wurde die deutsche Übersetzung in der DMW publiziert [1] und ist im Internet frei zugänglich (www.consortstatement.org/index.aspx?0=1335). Weitere Leitlinien z.B. für diagnostische Studien [2] und Beobachtungsstudien [3] folgten. Um die gemeinsamen Ziele zu fördern und ein einheitliches Informationsangebot zu schaffen, arbeiten die Initiativen seit kurzem im EQUATOR Network zusammen [4]. Dessen Webseite (www.equator-network.org) gibt einen Überblick über alle Berichtsleitlinien und ist eine erste Anlaufstelle für Interessierte.

Mit dem PRISMA-Statement wurde nun eine vollständig überarbeitete Empfehlung für die Publikation von systematischen Übersichtsarbeiten (Systematic Reviews) veröffentlicht. Es ist kürzlich gleichzeitig in vier internationalen Zeitschriften (PLoS Med, Ann Intern Med, BMJ, J Clin Epidemiol) erschienen und löst das 1999 veröffentlichte QUOROM-Statement ab. Dessen Akronym hatte immer wieder zu Schreibfehlern und Verwirrung geführt. Dem Modell anderer Initiativen folgend, wird in der PRISMA-Kurzversion eine Checkliste mit knappen Erklärungen angeboten [5]. Die 27 Punkte der Checkliste folgen der klassischen Einteilung von Fachartikeln in Titel, Abstrakt, Einführung, Methoden-, Ergebnisteil, Diskussion und Angaben über Studienfinanzierung. Dadurch ist die Orientierung für den forschungsversierten Leser sehr leicht. Die ausführliche Version [6] bietet darüber hinaus eine Fülle von Erklärungen und von Beispielen guter Berichterstattung. Sie vermittelt, quasi nebenbei, die einzelnen Schritte einer systematischen Übersichtsarbeit. Dies geschieht zwar immer aus der
Perspektive der Publikation, liefert aber einen wertvollen Beitrag zur anhaltenden Diskussion über methodische Brennpunkte bei der Erstellung von Übersichtsarbeiten. So werden etwa neue Entwicklungen zum Thema „Study Quality and Risk of Bias“ brandaktuell, knapp und präzise dargestellt. Die PRISMA-Initiative hat eine eigene Webseite (www.prisma-statement.org), von der Nutzer die Checkliste und auch ein hilfreiches Flussdiagramm als Vorlage herunterladen können.

Das PRISMA-Statement richtet sich in erster Linie an die unmittelbar Beteiligten des Publikationsprozesses, also an die Autoren, Fachgutachter und Herausgeber wissenschaftlicher Zeitschriften, in denen systematische Übersichtsarbeiten erscheinen. Natürlich wird damit auch den Lesern ein sehr gutes Instrument geboten; können sie doch anhand der Checkliste sehr schnell überprüfen, welche Detailinformationen von den Autoren berichtet werden und welche nicht. Die Leitlinie ist nicht nur für Übersichtsarbeiten im engeren Sinne hilfreich. Sie ist zudem nützlich für Autoren und Leser anderer Publikationen, in denen Ergebnisse aus patientenorientierten Studien systematisch zusammengeführt und ausgewertet werden (z.B. klinische Leitlinien, HTAReports). Denn auch solche Arbeiten folgen dem methodischen Grundgerüst einer systematischen Übersichtsarbeit, um den Qualitätsansprüchen der evidenzbasierten Medizin gerecht zu werden. Nicht zuletzt ist das PRISMA-Statement auch anwendbar auf die Synthese von Studienergebnissen, wenn diese aus Studien mit unterschiedlichem Design wie z.B. aus randomisierten und Beobachtungsstudien stammen. Damit gewinnt die Empfehlung eine gewisse Universalität. Dies macht sie für all diejenigen nützlich, die ein Interesse an hochwertiger und zuverlässiger medizinischer Information haben.

\section{Literatur}

1 Moher D, Schulz KF, Altman D. Das CONSORT Statement: Überarbeitete Empfehlungen zur Qualitätsverbesserung von Reports randomisierter Studien im Parallel-Design. Dtsch Med Wochenschr 2004; 129: T16-T20

2 Bossuyt PM, Reitsma JB, Bruns DE et al. Towards complete and accurate reporting of studies of diagnostic accuracy: The STARD Initiative. Ann Intern Med 2003; 138: 40-44

3 Elm E von, Altman DG, Egger $M$ et al. Das Strengthening the Reporting of Observational Studies in Epidemiology (STROBE-) Statement. Leitlinien für das Berichten von Beobachtungsstudien. Internist 2008; 49: 688-693

4 Altman DG, Simera I, Hoey J et al. EQUATOR: reporting guidelines for health research. Lancet 2008; 371: $1149-1150$

5 Moher D, Liberati A, Tetzlaff J et al. Preferred reporting items for systematic reviews and meta-analyses: the PRISMA statement. PLoS Med 2009; 6: e1000097

6 Liberati A, Altman DG, Tetzlaff J et al. The PRISMA statement for reporting systematic reviews and meta-analyses of studies that evaluate healthcare interventions: explanation and elaboration. BMJ 2009; 339: b2700

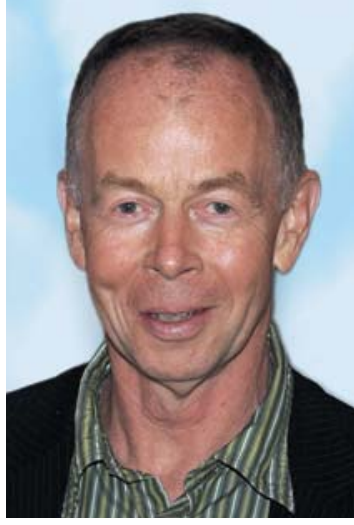

Dr. G. Antes

G. Antes ${ }^{1}$

E. von Elm 1,2

Medizinisches Publizieren

Schlüsselwörter

Systematische

Übersichtsarbeit

PRISMA Statement

QUOROM Statement

Evidenzbasierte Medizin

Keywords

Systematic review

PRISMA Statement

QUOROM Statement

Evidence-based medicine

Institut

1Deutsches Cochrane

Zentrum, Universitätsklinikum

Freiburg i. Brsg.

2Schweizer Paraplegiker

Forschung, Nottwil / Schweiz

Bibliografie

Dol 10.1055/s-0029-1233989

Dtsch Med Wochenschr 2009;

134: 1619 . (c) Georg Thieme

Verlag KG Stuttgart · New York .

ISSN 0012-0472

Korrespondenz

Dr. Gerd Antes

Deutsches Cochrane Zentrum Institut für Medizinische

Biometrie und Medizinische Informatik

Universität Freiburg

Stefan-Meier-Str. 26

79104 Freiburg

Tel. 0761/203-6706

Fax 0761/203-6712

eMail antes@cochrane.de

www.cochrane.de 